\title{
Simplifying Minimum Dose Electron Tomography
}

\author{
Robin T. Harmon, ${ }^{*}$ Steffen Meyer,* Christopher R. Booth* and Jacob Wilbrink* \\ * Gatan Research and Development, 5794 W. Las Positas Blvd, Pleasanton, CA 94588
}

Electron tomography is an important tool for electron microscopists trying to understand the 3dimensional structure of their specimens. Many TEM specimens, particularly biological ones, are sensitive to the total electron dose used during imaging. Several groups have demonstrated procedures for minimizing the dose during electron tomography $[1,2,3,4]$. Here we describe an approach to minimizing the electron dose for use with the Gatan TEM Tomography Software [5]. By offsetting the tracking areas from the data acquisition area the electron dose on the specimen is reduced by a factor of 4 . In addition to the low-dose capability, this solution is supported on a broad range of microscopes from different vendors, is fully integrated into the Gatan Microscopy Suite, and benefits from the image processing capabilities and other tools that this provides.

The acquisition of a tomographic tilt series would be straightforward were it not for the mechanical limitations of a typical microscope stage. As a specimen is tilted from one angle to the next the goniometer imposes both random and systematic shifts in $\mathrm{X}, \mathrm{Y}$, and $\mathrm{Z}$ on the specimen. These shifts can cause a loss of focus or lose the field of interest after a small number of tilts, depending on the magnification and the properties of the given stage.

Gatan's tomography software allows automated and pre-calibration based methods to correct for stage errors [1]. In the automated method, drift in the X-Y plane is measured by comparing an image from the current tilt angle to a reference image. To measure the drift in the $\mathrm{Z}$ direction the beam-tilt induced image-shift method is used [6].

Here we describe an extension to the Gatan TEM Tomography Software that allows the user to define regions for automated $\mathrm{X} / \mathrm{Y}$ and $\mathrm{Z}$ tracking that are displaced along the tilt axis from the data acquisition area. Each of the tracking regions and the data acquisition area can have different illumination conditions (spot size, brightness). The electron dose in the tracking regions can be increased over that in the data acquisition area in order to improve the signal-to-noise ratio of the images used in determining the drift and hence improve the reliability of these measurements.

A graphical user interface is provided to help set up the automated tracking regions. The user adjusts the relative offsets of the two tracking regions from the data acquisition region by means of sliders (Figure 1) and can record the illumination conditions to be used at each of the 3 regions. The software assists the user by constraining the region's offset to lie on the tilt axis and allows the user to preview a sample image from any of the 3 regions (Figure 2).

For users with electron-dose sensitive specimens the ability to acquire tracking data from regions other than the area of interest should enable acquisition of tilt series from specimens that would otherwise suffer too much damage to be useful or track with a higher signal-to-noise ratio than before while still reducing the overall electron dose on the area of interest. 
References

[1] D. N. Mastronarde., Journal of Structural Biology (2005) 152:36-51.

[2] B. K. Rath et al., Journal of Structural Biology (1997) 120:210-218.

[3] S. Nickell, Journal of Structural Biology (2005) 149:227-234.

[4] Q.S. Zheng, Journal of Structural Biology (2004) 147:91-101.

[5] http://www.gatan.com/products/software/tomography.php "3D Tomography-Acquisition

Software".

[6] A.J. Koster et al., Electron Microscopy (1992) 1: 119-123.
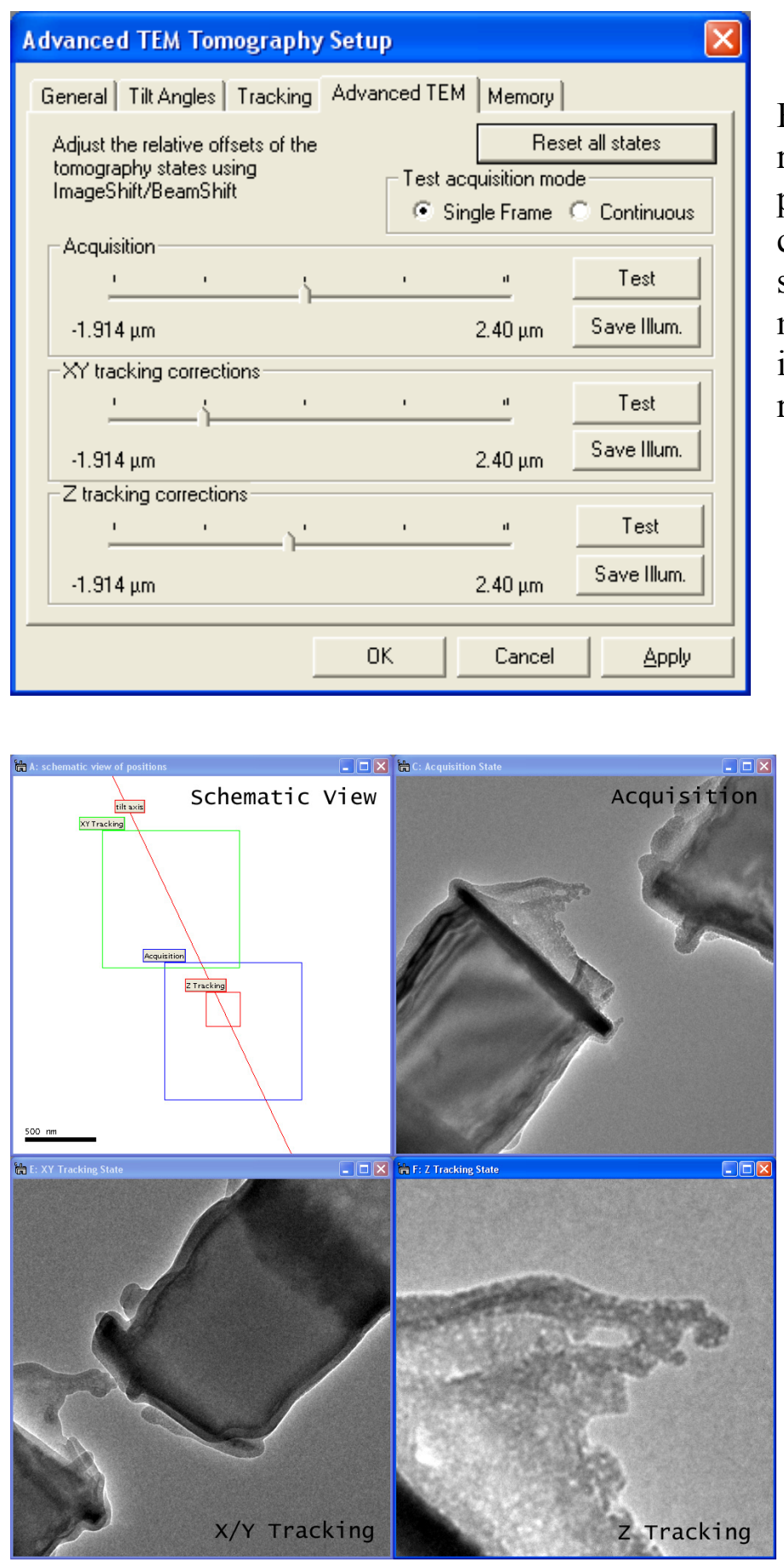

FIG. 1. In this panel the user can set up the relative offsets of the $\mathrm{X} / \mathrm{Y}$ and $\mathrm{Z}$ tracking positions from the data acquisition region. The conditions at each location can be tested with a single image acquisition ('Test' button) or monitored in a continually updating view. The illumination conditions can be set for each region independently.

FIG. 2. As the user adjusts the relative offsets of the tracking and data acquisition regions the 'Schematic View' updates to show the regions relative positions. At any time the user can view sample images from each of the regions (labeled 'Acquisition', ' $\mathrm{X}-\mathrm{Y}$ Tracking' and ' $\mathrm{Z}$ Tracking' in the figure). 